\title{
Nomogram for predicting radiation maculopathy in patients treated with Ruthenium-106 plaque brachytherapy for uveal melanoma
}

\author{
Luca Tagliaferri, MD, PhD!, Monica Maria Pagliara, MD², Carlotta Masciocchi ${ }^{3}$, Andrea Scupola, MD², \\ Luigi Azario4 , Gabriela Grimaldi, MD², Rosa Autorino, MD, PhD', Maria Antonietta Gambacorta, MD, PhD³, \\ Antonio Laricchiuta!, Luca Boldrini, MD³, Vincenzo Valentini, MD³, Maria Antonietta Blasi, MD5 \\ IPolo Scienze Oncologiche ed Ematologiche, Università Cattolica del Sacro Cuore, Fondazione Policlinico Universitario Agostino Gemelli, Roma, \\ 2Polo Scienze dell'invecchiamento, neurologiche, ortopediche e della testa-collo, Università Cattolica del Sacro Cuore, Fondazione Policlinico \\ Universitario Agostino Gemelli, Roma, ${ }^{3}$ Polo Scienze Oncologiche ed Ematologiche, Istituto di Radiologia, Università Cattolica del Sacro \\ Cuore, Fondazione Policlinico Universitario Agostino Gemelli, Roma, ${ }^{4}$ Polo scienze delle immagini, di laboratorio e infettivologiche, Istituto \\ di Fisica, Università Cattolica del Sacro Cuore, Fondazione Policlinico Universitario Agostino Gemelli, Roma, ${ }^{5}$ Polo Scienze dell'invecchiamento, \\ neurologiche, ortopediche e della testa-collo, Istituto di Oftalmologia, Università Cattolica del Sacro Cuore, Fondazione Policlinico Universitario \\ Agostino Gemelli, Roma, Italy
}

\begin{abstract}
Purpose: To develop a predictive model and nomogram for maculopathy occurrence at 3 years after ${ }^{106} \mathrm{Ru} /{ }^{106} \mathrm{Rh}$ plaque brachytherapy in uveal melanoma.

Material and methods: Clinical records of patients affected by choroidal melanoma and treated with ${ }^{106} \mathrm{Ru} /{ }^{106} \mathrm{Rh}$ plaque from December 2006 to December 2014 were retrospectively reviewed. Inclusion criteria were: dome-shaped melanoma, distance to the fovea $>1.5 \mathrm{~mm}$, tumor thickness $>2 \mathrm{~mm}$, and follow-up $>4$ months. The delivered dose to the tumor apex was 100 Gy. Primary endpoint of this investigation was the occurrence of radiation maculopathy at 3 years. Analyzed factors were as follows: gender, age, diabetes, tumor size (volume, area, largest basal diameter and apical height), type of plaque, distance to the fovea, presence of exudative detachment, drusen, orange pigment, radiation dose to the fovea and sclera. Univariate and multivariate Cox proportional hazards analyses were used to define the impact of baseline patient factors on the occurrence of maculopathy. Kaplan-Meier curves were used to estimate freedom from the occurrence of the maculopathy. The model performance was evaluated through internal validation using area under the ROC curve (AUC), and calibration with Gronnesby and Borgan tests.

Results: One hundred ninety-seven patients were considered for the final analysis. Radiation-related maculopathy at 3 years was observed in 41 patients. The proposed nomogram can predict maculopathy at 3 years with an AUC of 0.75. Distance to fovea appeared to be the main prognostic factor of the predictive model (hazard ratio of 0.83 [0.76-0.90], $p<0.01$ ). Diabetes (hazard radio of 2.92 [1.38-6.20], $p<0.01$ ), and tumor volume (hazard radio of 21.6 [1.66-281.14], $p=0.02$ ) were significantly predictive for maculopathy occurrence. The calibration showed no statistical difference between actual and predicted maculopathy $(p=1)$.

Conclusions: Our predictive model, together with its nomogram, could be a useful tool to predict the occurrence of radiation maculopathy at 3 years after the treatment.

Key words: brachytherapy, maculopathy, nomogram, ruthenium plaque, radiotherapy, uveal melanoma.

\section{Purpose}

Uveal melanoma is an uncommon cancer, affecting from 6 to 7 individuals per million per year [1]. The Collaborative Ocular Melanoma Study (COMS) proved plaque brachytherapy to be a reliable alternative to surgical enu- cleation for the treatment of medium-sized choroidal melanomas. The study demonstrated equivalent rates of local control and survival between plaque brachytherapy and enucleation $[2,3,4,5,6,7,8,9,10]$. Applicators loaded with ${ }^{125}$ I have been used in the COMS study [2], and also for
Address for correspondence: Carlotta Masciocchi, Polo Scienze Oncologiche ed Ematologiche, Istituto di Radiologia, Università Cattolica del Sacro Cuore, Fondazione Policlinico Universitario Agostino Gemelli, Largo Francesco Vito 1, 00168 Roma, Italia, phone: +39393 9913958, e-mail: carlotta.masciocchi@unicatt.it
Received: 22.08.2017 Accepted: 06.11.2017

Published: 30.12.2017 
this reason, the use of such applicators has become the primary choice for uveal melanoma brachytherapy in North America.

Nevertheless, many European institutions have gained experience using ${ }^{106} \mathrm{Ru} /{ }^{106} \mathrm{Rh}$, a beta emitter, mainly for the treatment of small and medium-sized choroidal melanomas, reporting comparable outcomes in terms of overall survival [11]. ${ }^{106} \mathrm{Ru} /{ }^{106} \mathrm{Rh}$ is a beta emitter with more restricted range in comparison to gamma sources, and offers some advantages with respect to ${ }^{125} \mathrm{I}$, such us a better radiation protection for the operators and less side effects for adjacent healthy tissues. Excellent local control outcomes have been reported by a number of groups using ${ }^{106} \mathrm{Ru} /{ }^{106} \mathrm{Rh}$ plaques for small-medium sized uveal melanoma with acceptable rates of radiation-induced complications $[12,13,14]$. Naseripour et al. have also shown that ${ }^{106} \mathrm{Ru} /{ }^{106} \mathrm{Rh}$ brachytherapy is a successful alternative to enucleation for thick uveal melanomas: lower doses of radiations to tumor apex, assured that enough dose is delivered to the sclera, can successfully treat such tumors, probably as a consequence of the effects of radiation on tumor blood supply [15]. Furthermore, a dosimetric comparison of ${ }^{125} \mathrm{I}$ versus ${ }^{106} \mathrm{Ru} /{ }^{106} \mathrm{Rh}$ plaques demonstrated that ${ }^{106} \mathrm{Ru} /{ }^{106} \mathrm{Rh}$ plaques can provide adequate dose coverage to small tumors, sparing critical nearby structures more effectively than ${ }^{125} \mathrm{I}$ [16]. Dose distribution comparison between ${ }^{125} \mathrm{I}$ and ${ }^{106} \mathrm{Ru} /{ }^{106} \mathrm{Rh}$ is reported in Figure 1. However, brachytherapy with ${ }^{106} \mathrm{Ru} /{ }^{106} \mathrm{Rh}$ plaques is not free from local toxicity, and the benefits of saving the eye may be reduced by visual function impairment, secondary to radiation-induced toxicity (such as cataract, optic neuropathy or retinopathy) [17].

Radiation maculopathy appears to be the most common complication, although several effective therapy approaches for the prevention and treatment of this disease are available $[18,19]$. A radiation induced maculopathy predictive model could be a useful tool in order to identify the patients that could take more advantage from pre- ventive strategies $[20,21]$. Primary aim of this study is to develop a nomogram for the prediction of such complication in patients affected by uveal melanoma who underwent ${ }^{106} \mathrm{Ru} /{ }^{106} \mathrm{Rh}$ brachytherapy.

\section{Material and methods}

For this analysis, we considered all consecutive patients who underwent ${ }^{106} \mathrm{Ru} /{ }^{106} \mathrm{Rh}$ plaque brachytherapy for uveal melanoma at the Gemelli Advanced Radiation Therapy Center from December 2006 to December 2014. Clinical and therapy data were collected from the hospital multidivisional electronic database, and were electronically elaborated with the COBRA-Storage System (C-SS) to ensure patient's privacy $[22,23]$.

COBRA (Consortium for Brachytherapy Data Analysis) provides a set of ontologies expressed in the Web Ontology Language (OWL), and defined as "COBRA-ONT" to describe and share knowledge in an intelligent meeting-room environment.

COBRA-ONT has been used for the setup of our database with an open architecture that forecasts future data integration with databases originating from other institutions, creating a shared-data environment to enlarge patients' sample and better validate current results.

Institutional review board and ethics committee approval for using data collecting software had been obtained. Each patient was evaluated by a multidisciplinary tumor board, including ophthalmologists mainly involved in ocular oncology, radiation oncologists, clinical oncologists, and medical physicists [24].

All patients underwent complete ophthalmic exam during the first visit and the successive follow-up contacts. Slit lamp biomicroscopy, ophthalmoscopy with fundus photography, and A-B scan ultrasonography were routinely performed. Patient's demographic characteristics were collected, including age, gender, and medi-
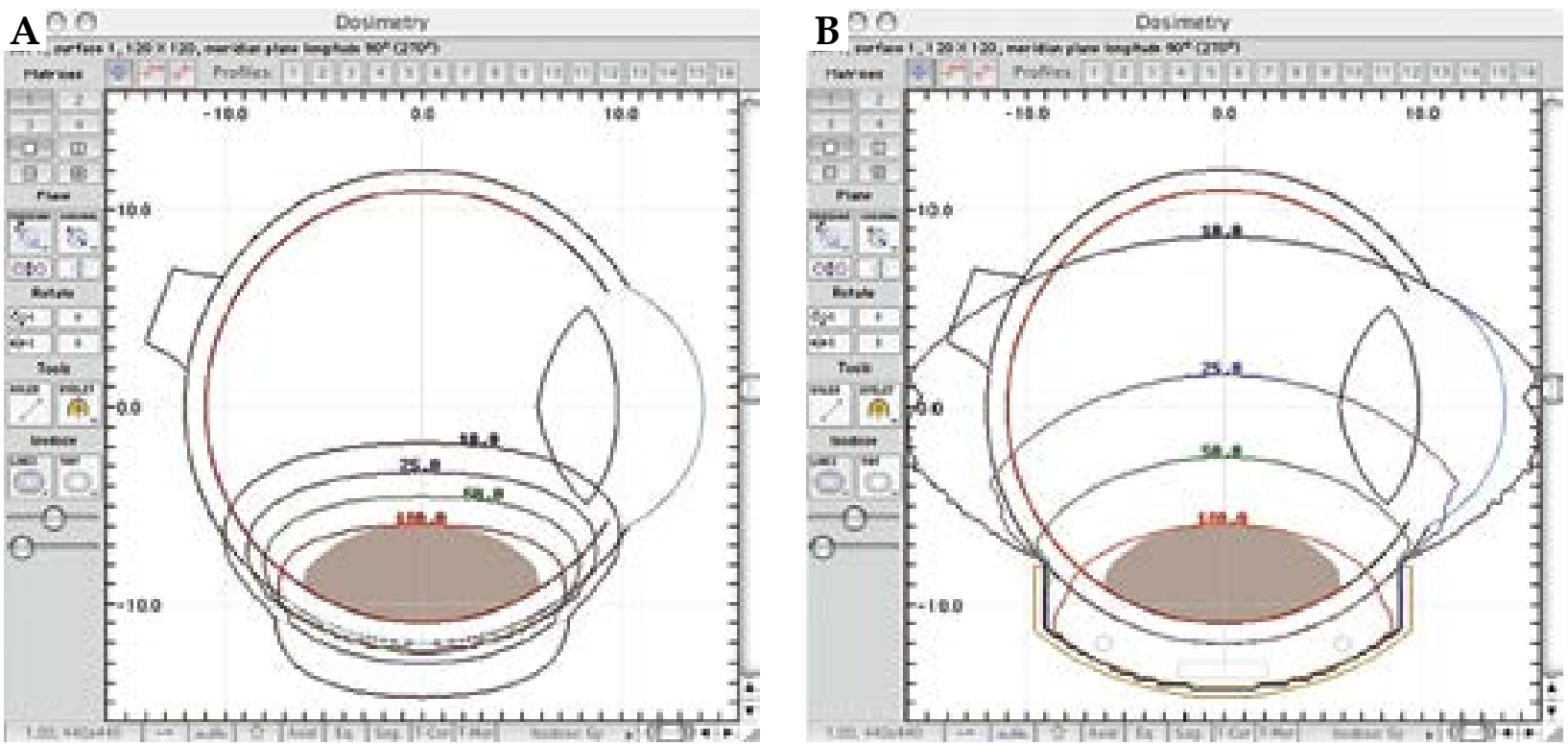

Fig. 1. Comparison of dose distribution for ${ }^{106} \mathrm{Ru} /{ }^{106} \mathrm{Rh}(\mathrm{A})$ and ${ }^{125} \mathrm{I}$ plaques (B). Dose normalized to apex of the tumor and expressed in percentage 
Table 1. Summary patient's and tumor characteristics $(N=197)$

\begin{tabular}{lc} 
Factor & $n$ \\
\hline Age & 68 \\
\hline Median & $57-74$ \\
\hline Quartile (first-third quartile) & \\
\hline Gender & $87(44.15 \%)$ \\
\hline Male & $110(55.83 \%)$ \\
\hline Female & $25(12.69 \%)$ \\
\hline Diabetes & $172(87.30 \%)$ \\
\hline Yes & \\
\hline No
\end{tabular}

\begin{tabular}{lc}
\hline Hypertension & \\
\hline Yes & $94(47.71 \%)$ \\
\hline No & $103(52.28 \%)$ \\
\hline
\end{tabular}

\begin{tabular}{lc}
\hline Tumor staging* & \\
\hline $\mathrm{T} 1$ & $87(44.16 \%)$ \\
\hline $\mathrm{T} 2$ & $108(54.82 \%)$ \\
\hline $\mathrm{T} 3$ & $2(1.01 \%)$
\end{tabular}

T3 $2(1.01 \%)$

\begin{tabular}{|c|c|}
\hline \multicolumn{2}{|l|}{ Largest basal diameter (mm) } \\
\hline Median & 10.10 \\
\hline Quartile (first-third quartile) & $8.41-11.87$ \\
\hline \multicolumn{2}{|l|}{ Tumor height (mm) } \\
\hline Median & 3.3 \\
\hline Quartile (first-third quartile) & $2.87-3.91$ \\
\hline \multicolumn{2}{|l|}{ Total dose to disc (Gy) } \\
\hline Median & 17.72 \\
\hline Quartile (first-third quartile) & $1.63-49.26$ \\
\hline \multicolumn{2}{|l|}{ Total dose to macula (Gy) } \\
\hline Median & 28.20 \\
\hline Quartile (first-third quartile) & $2.87-158$ \\
\hline \multicolumn{2}{|l|}{ Total dose to sclera (Gy) } \\
\hline Median & 268.50 \\
\hline Quartile (first-third quartile) & $232.8-351.8$ \\
\hline \multicolumn{2}{|l|}{ Volume (ml) } \\
\hline Median & 0.19 \\
\hline Quartile (first-third quartile) & $0.13-0.28$ \\
\hline \multicolumn{2}{|l|}{ Distance to lens (mm) } \\
\hline Median & 17.26 \\
\hline Quartile (first-third quartile) & $14.79-18.45$ \\
\hline
\end{tabular}

${ }^{*}$ AJCC seventh edition cal history. The presence of maculopathy was recorded at baseline and during each further follow-up visit, through a complete eye examination and optical coherence tomography (OCT).

The considered tumor data included anatomical location (choroidal, ciliary, cilio-choroidal, irido-ciliary, or irido-cilio-choroidal), location of tumor epicenter (superior, inferior, nasal, temporal, or posterior pole), tumor size (basal dimension and thickness evaluated with A-B scan ultrasonography), and tumor volume, calculated with treatment a planning system (TPS).

Dosimetry for brachytherapy was performed using a dedicated 3D calculation software (Plaque Simulator ${ }^{\mathrm{TM}}$, Bebig, Berlin, Germany) [25].

The prescribed dose to the tumor apex was $100 \mathrm{~Gy}$, and an extra safety margin of $1 \mathrm{~mm}$ was added to the diameters of tumor. Target geometry was discussed and validated in all cases together with ocular oncologists. Treatment approval criteria were 100 Gy dose delivered at tumor apex, with the entire volume receiving at least $95 \%$ of the prescribed dose. Four types of ${ }^{106} \mathrm{Ru} /{ }^{106} \mathrm{Rh}$ plaques (Bebig, Germany) of different sizes (Models CCA, CCD, CCB, COB with nominal diameters of $15.5 \mathrm{~mm}, 17.8 \mathrm{~mm}, 20 \mathrm{~mm}$, and $20 \mathrm{~mm}$, respectively) were available for treatment delivery. Patients inclusion criteria were: dome-shaped melanoma, distance to the fovea $>1.5 \mathrm{~mm}$, tumor thickness $>2 \mathrm{~mm}$, and follow-up $\geq 4$ months.

According to the INTERACTS (INTErventional Radiotherapy ACtive Teaching School) guidelines for quality assurance in ocular melanoma brachytherapy, tumor localization is firstly performed using transillumination or indirect ophthalmoscopy. Tumor margins are then marked on the sclera with a surgical marking pen, and a dummy applicator is sutured in the appropriate position. The loaded plaque will replace the dummy applicator, and an ultrasound check will be postoperatively performed to verify the accuracy of plaque positioning.

Follow-up examinations were planned fifteen days after surgery, then every four months for the first year; every six months for the following five years, and then annually. Ophthalmoscopy, slit lamp biomicroscopy, A-B scan ultrasonography, and fundus photography were repeated during each follow-up visit.

A screening for secondarisms has been performed every six months with liver function tests and imaging (ultrasonography). The following covariates were considered for our analysis: gender, age, diabetes, tumor dimensional parameters (size, volume, area, largest basal diameter, and apical height), type of plaque, distance to the fovea, presence of exudative detachment, drusen or orange pigment presence, and radiation dose to fovea and sclera.

Kaplan-Meier analysis with log-rank test [26] and univariate Cox proportional hazards model at 3 years were performed to identify the covariates, which may have an effect on the outcome of maculopathy. Multivariate analysis was performed using Cox proportional hazard model and hazard ratios (HR) were calculated from the model coefficients for each covariate. A $p$-value $\leq 0.05$ was considered significant. The stepwise Akaike's information 
criterion (AIC) selection method was applied to select the best final model [27], and discrimination power was evaluated using AUC of model (area under the curve) [28]. The time independence of predictors (Cox assumption) was verified using Schoenfeld residuals [29].

A linear cross-correlation matrix (Pearson's $\chi^{2}$ test) was used to assess the presence of interactions among predictors: the statistical power of the model increases when no interactions among the predictors are observed. A calibration plot was also elaborated in order to estimate the accuracy of the model using Gronnesby and Borgan goodness-of-fit (GOF) test: $p$-values $<0.05$ indicate lack of fit of the model.

Finally, the entire dataset has been used for internal validation employing a resampling technique known as bootstrapping (TRIPOD type $1 \mathrm{~b}$ internal validation [30]) in order to evaluate the performance of the developed model. In particular, 1,000 datasets with the same size of the primary patients dataset have been generated from the original one by random sampling with replacement.

The AUC value has been then calculated for each dataset, and the final AUC represents their median value. A nomogram was then realized as tool to visualize the output of our predictive model.

All statistical analyses have been performed using $\mathrm{R}$ version 3.3.1 [31].

\section{Results}

Based on inclusion criteria, 202 patients were selected from our database. A minimal plaque misplacement was observed in 5 cases during the follow-up visits, with the actinic scar showing to be not overlapping with the tumor borders. These cases were consequently excluded from our statistical analysis, as they had a pre-treatment plan different from the delivered one.

All the patients with misplaced plaque required adjuvant transpupillary thermotherapy (TTT) and are currently alive with no evidence of distant metastases. For

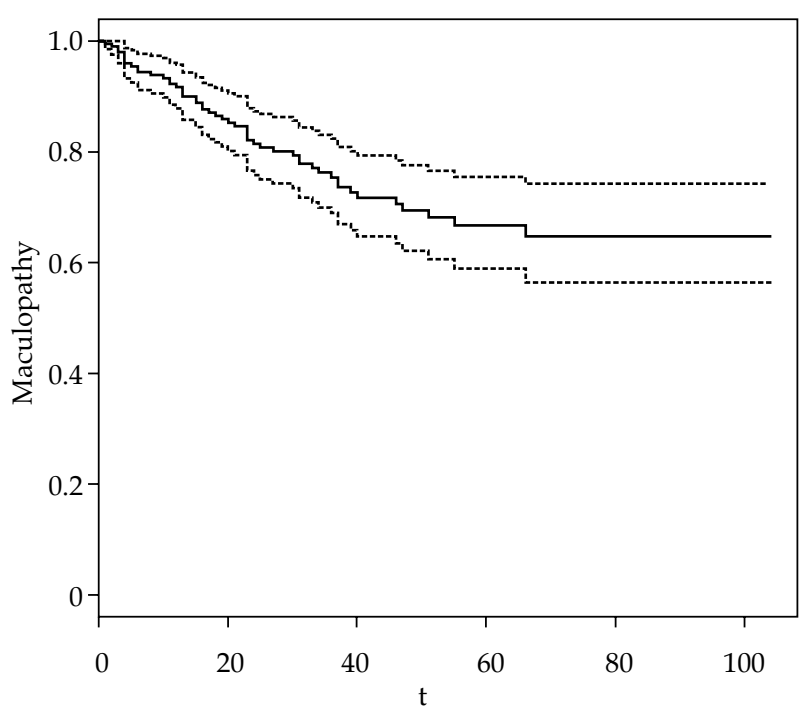

Fig. 2. Kaplan-Meier curve of maculopathy (solid line) with confidence interval (dashed line)

one of them, re-treatment brachytherapy was required, while two patients underwent proton beam radiotherapy. In total, 197 patients with a median age of 68 years (range, 17-92) were therefore considered for this analysis. The median age, age range, gender, medical history, and tumor staging [32], pathologic and dosimetric characteristics are reported in Table 1 . The median of the follow-up was 51 months (range, 4-107 months).

Maculopathy at 3 years occurred in 41 patients (21\%). The Kaplan-Meier curve is shown in Figure 2. ${ }^{106} \mathrm{Ru} /{ }^{106} \mathrm{Rh}$ plaques were used (Bebig, Germany) CCA in 66 cases, COB in 39 cases, CCB in 50 cases, and CCD in 42 cases. Fifty eight patients $(29.7 \%)$ underwent transpupillary thermotherapy (TTT) as adjuvant therapy, mainly in case of juxtapapillary tumors.

Thirteen patients $(6.5 \%)$ died during follow-up period (4 deaths were attributed to metastatic uveal melanoma,

Table 2. Univariate and multivariate analysis for predicting the occurrence of maculopathy at 3 years

\begin{tabular}{|c|c|c|c|c|}
\hline \multirow[t]{2}{*}{ Factors } & \multirow{2}{*}{$\frac{\text { Univariate analysis }}{p \text {-value }}$} & \multicolumn{3}{|c|}{ Multivariate analysis } \\
\hline & & Hazard ratio & $95 \% \mathrm{Cl}$ & $p$-value \\
\hline Retinal detachment & $0.01^{*}$ & - & - & 0.06 \\
\hline Diabetes & $0.02^{*}$ & 2.92 & $1.38-6.20$ & $<0.01^{*}$ \\
\hline Tumor thickness (mm) & $0.03^{*}$ & - & - & 0.53 \\
\hline Distance from fovea $(\mathrm{mm})$ & $<0.01^{*}$ & 0.83 & $0.76-0.90$ & $<0.01^{*}$ \\
\hline Dose to fovea (Gy) & $<0.01^{*}$ & - & - & 0.80 \\
\hline Dose to optic disk (Gy) & $<0.01^{*}$ & - & - & 0.65 \\
\hline Volume $(\mathrm{ml})$ & $0.03^{*}$ & 21.61 & $1.66-281.14$ & $0.02^{*}$ \\
\hline Distance from optic nerve (mm) & $<0.01^{*}$ & - & - & 0.15 \\
\hline Distance from lens (mm) & $<0.01^{*}$ & - & - & 0.86 \\
\hline
\end{tabular}

*Statistical significant: $p$-value $<0.05$ 
A

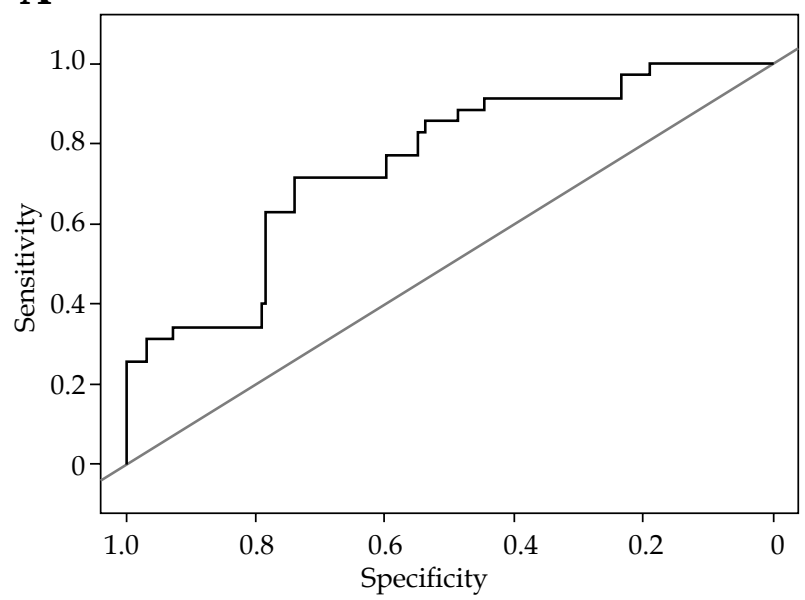

B

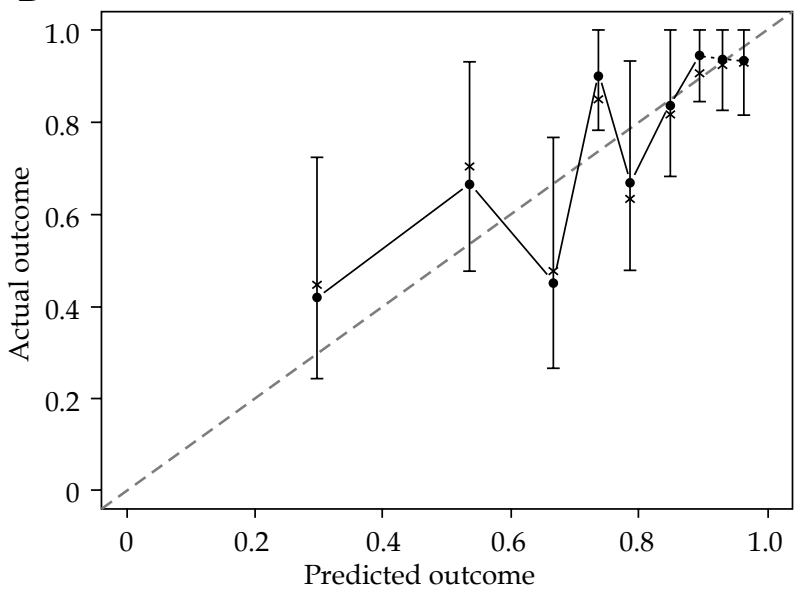

Fig. 3. A) Receiver operating characteristic (ROC) curve of the multivariate Cox proportional hazard model based on dataset population. Area under the curve (AUC) is $0.75(\mathrm{CI}=0.67-0.83)$. B) Internal validation: calibration plot estimating the observed probabilities of the outcome Maculopathy = TRUE ( $y$-axis) in relation to the predicted probabilities ( $x$-axis). Dotted line indicates perfect prediction. Round black dots show subjects grouped by similar probabilities (quantiles) of dataset population, the brackets show $95 \%$ CI of the prediction, and the points are joint by straight lines. No statistically significant difference was observed between actual and predicted maculopathy $(p=1)$

while the remaining 9 deaths resulted to be unrelated to it). Thirteen patients $(6.5 \%)$ had local failure: after evidence of disease recurrence, one patient required transcleral resection, two patients were re-treated with brachy- therapy, eight patients underwent enucleation, one patient was treated with transpupillary thermotherapy, and two patients died due to metastatic disease. The subset of univariate significant variables related to the devel-

Points

Volume

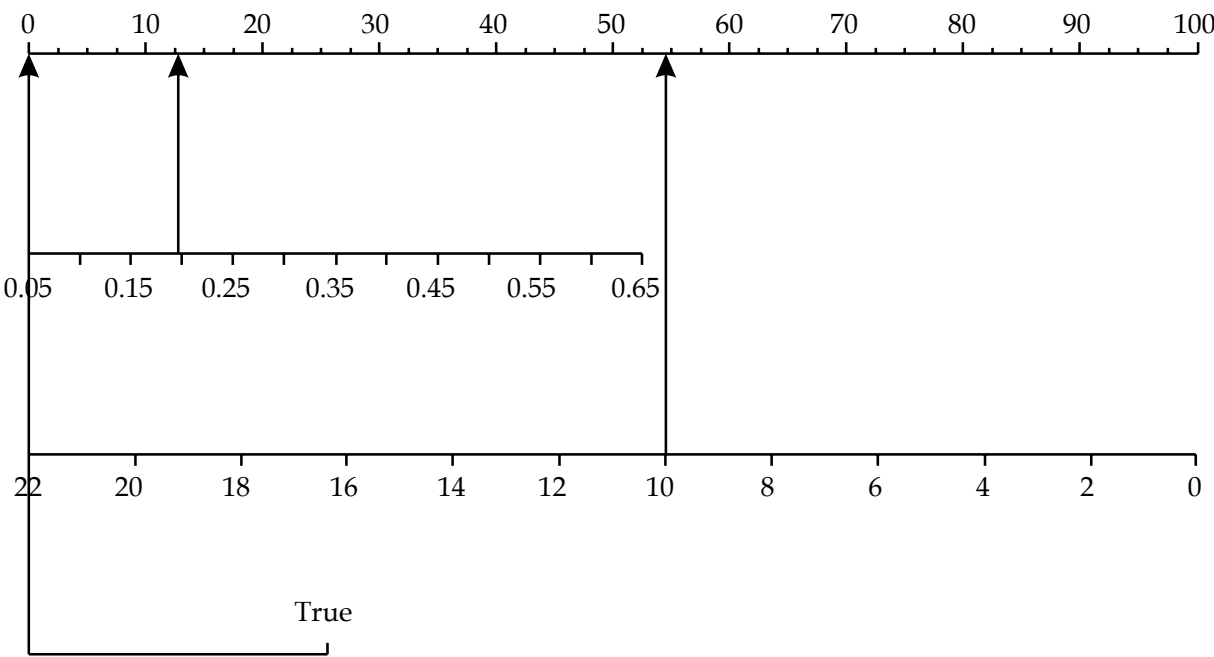

Diabetes

False

Total points

$$
0
$$$$
20
$$$$
40
$$

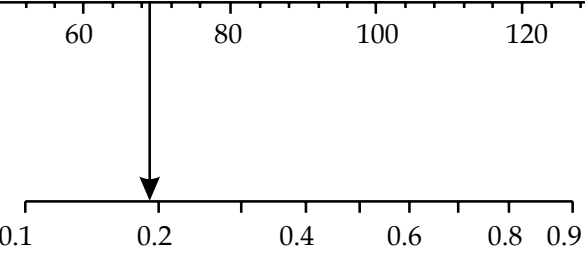

Predicted value

0.1

0.2

Fig. 4. Nomogram using example 1: a woman with a tumor volume of $0.2 \mathrm{ml}$, tumor distance to fovea of $10 \mathrm{~mm}$, and not affected by diabetes, would have 68 total points $(13+55+0)$, corresponding to a $19 \%$ probability of developing maculopathy at 3 years after the treatment 


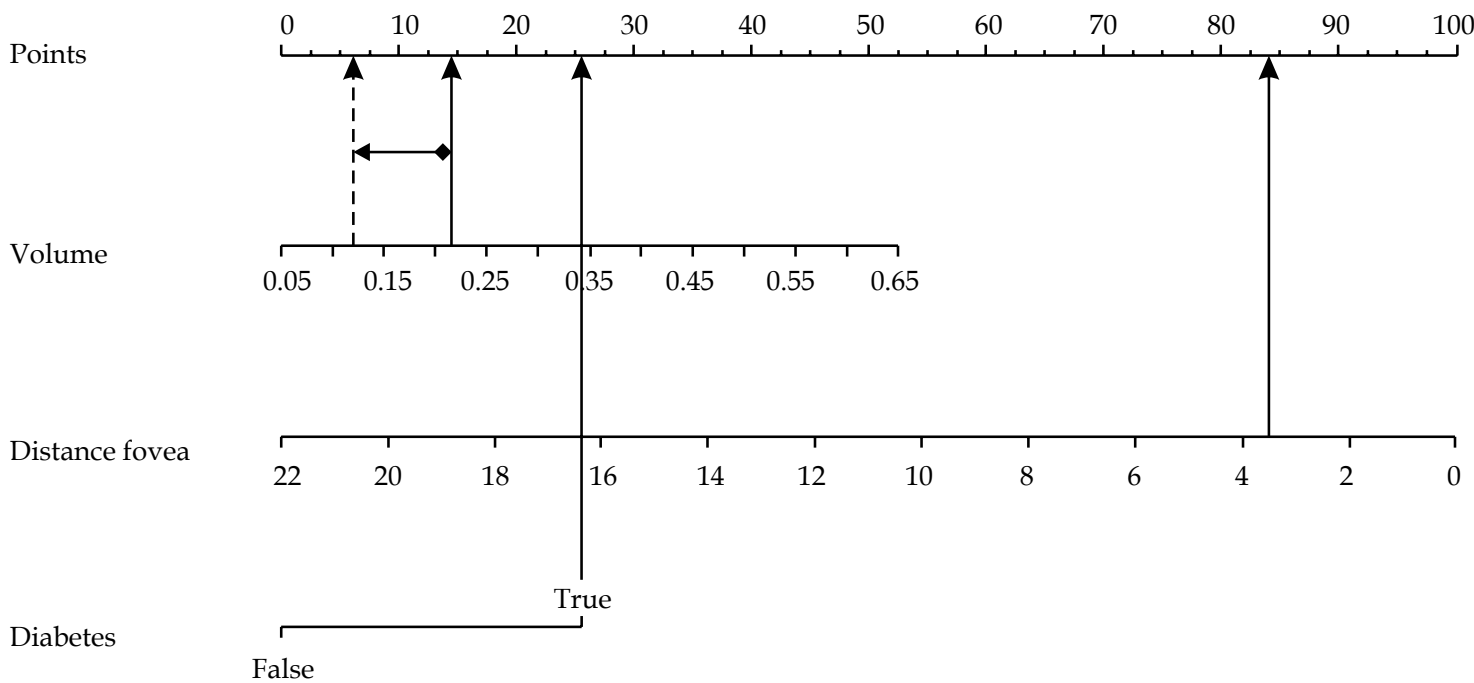

Total points

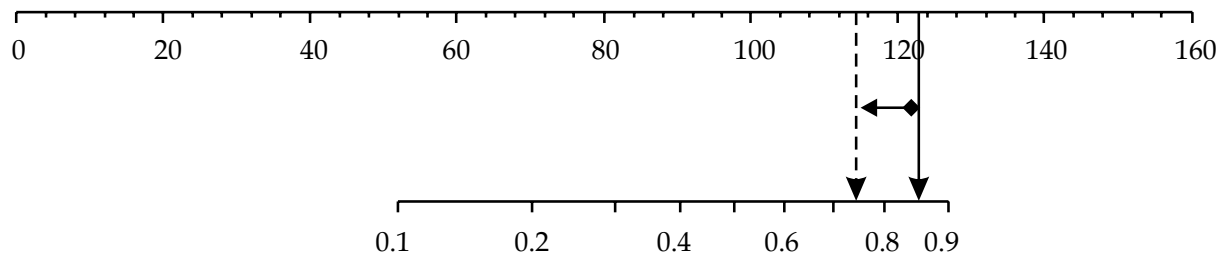

Predicted value

Fig. 5. Nomogram using example 2: patient extracted from the database of the photodynamic therapy (PDT) experience. This patient had diabetes, a tumor thickness of $2.79 \mathrm{~mm}$, tumor volume of $0.22 \mathrm{ml}$, and tumor distance to fovea of $3.83 \mathrm{~mm}$ before neoadjuvant PDT. After PDT, a reduction of tumor thickness (from $2.79 \mathrm{~mm}$ to $2.25 \mathrm{~mm}$ ) and volume (from $0.22 \mathrm{ml}$ to $0.12 \mathrm{ml}$ ) were observed. The nomogram shows a probability decrease to develop maculopathy from $86 \%$ to $74 \%$ in the patient in exam. Patient's characteristics before PDT are indicated with solid line and after PDT with dashed line

opment of maculopathy is summarized in Table 2. Interestingly, patients with primary lesion at a distance to the fovea $>15.45 \mathrm{~mm}$ did not develop maculopathy in our dataset.

Plaque $\mathrm{CCB}$, plaque $\mathrm{CCD}$, plaque $\mathrm{COB}$, age, gender, presence of orange pigment, largest basal diameter, scleral dose, presence of drusen, area of the tumor, and tumor thickness were not associated with a statistically significant risk of developing radiation maculopathy.

By multivariate analysis, a decrease in the distance to the fovea (hazard ratio of 0.83 [0.76-0.90], $p<0.01$ ), an increase in tumor volume (hazard radio of 21.6 [1.66281.14], $p=0.02$ ) and the presence of diabetes (hazard radio of 2.92 [1.38-6.20], $p<0.01$ ) were found to be significant predictors of maculopathy (see Table 2).

The proportional hazards assumption for Cox model was respected, and no linear interactions among the predictors were observed. An AUC model of 0.75 (CI = 0.67-0.83) has been obtained (Figure 3A).

Nomogram tool was used to graphically represent the final model, and could be used in order to predict the occurrence of maculopathy at 3 years after the brachytherapy treatment. No statistically significant difference $(p=1)$ has been observed between actual and predicted maculopathy, and the corresponding calibration plot, as shown in Figure 3B.
The internal validation, using bootstrapping technique $(1,000$ random samples), confirmed the goodness of the model fit with an AUC of 0.75 .

\section{Discussion}

A homogeneous group of patients affected by uveal melanoma was selected for this investigation. Radiation induced retinopathy/maculopathy and optic neuropathy represent late-onset complications of brachytherapy and are characterized by a slowly progressive occlusive vasculopathy, which produces variable ischemic damages and leads to subsequent visual loss [33]. To the best of our knowledge, some predictive models about visual acuity have been developed in recent years, but none predicting maculopathy in patients affected by uveal melanoma and treated with ${ }^{106} \mathrm{Ru} /{ }^{106} \mathrm{Rh}$ plaque is currently available [34,35].

The original contribute of this study is the realization of a nomogram for maculopathy occurrence at 3 years after treatment.

Basing on patient and disease information generally available before treatment delivery, our model showed that the risk of maculopathy occurrence at 3 years can be best predicted by the following pool of covariates: reduced distance to the fovea [36], presence of diabetes, and 
higher tumor volume. As an example, a woman without diabetes affected by a tumor with a volume of $0.2 \mathrm{ml}$ and a distance to the fovea of $10 \mathrm{~mm}$ would have a probability of $19 \%$ to develop maculopathy at 3 years after the treatment (Figure 4).

The use of a decision support system (DSS) as the nomogram we propose, could be useful for determining patient's visual prognosis, and lead to overall risk of visual acuity loss reduction, allowing the best tailored treatment choice for each patient both in neoadjuvant [37] or adjuvant treatment settings [38]. Furthermore, by giving patients more information regarding their prognosis, our nomogram could help to better assess and understand the risks associated with this radiation therapy procedure during the informed consent discussion.

The performance of photodynamic therapy (PDT) as neoadjuvant treatment before brachytherapy for amelanotic choroidal melanoma have been evaluated in a previous experience [37]. PDT appears to reduce tumor thickness in $73.4 \%$ of cases with a mean reduction of $23.3 \%$. Consequently, a dose reduction of $19.3 \%$ and $27.1 \%$ to fovea and optic nerve, respectively, can be appreciated.

As a result, a significant decrease of irradiation side effects on visual function could be reached, without compromising disease control. Furthermore, our nomogram could be used to select patients affected by choroidal melanoma, who could benefit more from neoadjuvant treatments before brachytherapy, as tumor thickness appeared to be correlated with the disease volume (Figure 5). However, further studies are needed to better understand this correlation.

In addition, patients with a high risk of maculopathy development might be candidates for trials evaluating both preventive approaches or the direct treatment of this condition [38].

Since only $21 \%$ of patients is expected to develop radiation induced maculopathy, as demonstrated also in the present study, an accurate prediction model would help in identifying preventive treatments or support.

The main limitation of this study is represented by the fact that our nomogram has not been externally validated using an independent dataset originating from other institutions. Therefore, an external validation of the proposed model would be of great interest in order to confirm our observations on a more heterogeneous population through a reliable generalization process.

\section{Conclusions}

The nomogram, based on our radiation induced maculopathy predictive model, appears to be a useful tool for maculopathy occurrence prediction at 3 years after brachytherapy for uveal melanomas. The use of such DSS could help clinicians to reliably identify patients with higher risk of visual loss, and plan the best treatment for each of the patient on the basis of the characteristics of both the tumor and the single patient. Moreover, the nomogram can be introduced in daily clinical practice to enrich and better describe the informed consent procedures, offering patients more reliable prognostic information.

\section{Disclosure}

Authors report no conflicts of interest.

\section{References}

1. Singh AD, Topham A. Incidence of uveal melanoma in the United States: 1973-1997. Ophthalmology 2003; 110: 956-961.

2. Diener-West M, Earle JD, Fine SL et al.; Collaborative Ocular Melanoma Study Group. The COMS randomized trial of iodine 125 brachytherapy for choroidal melanoma, III: initial mortality findings. COMS Report No. 18. Arch Ophthalmol 2001; 119: 969-982.

3. Diener-West M, Hawkins BS, Markowitz JA et al. A review of mortality from choroidal melanoma. II. A meta-analysis of 5-year mortality rates following enucleation, 1966 through 1988. Arch Ophthalmol 1992; 110: 245-250.

4. Seddon JM, Gragoudas ES, Egan KM et al. Relative survival rates after alternative therapies for uveal melanoma. Ophthalmology 1990; 97: 769-777.

5. Augsburger JJ, Schneider S, Freire J et al. Survival following enucleation versus plaque radiotherapy in statistically matched subgroups of patients with choroidal melanomas: results in patients treated between 1980 and 1987. Graefes Arch Clin Exp Ophthalmol 1999; 237: 558-567.

6. Hungerford JL. Current trends in the treatment of ocular melanoma by radiotherapy. Clin Exp Ophthalmol 2003; 31: 8-13.

7. Seregard S. Long-term survival after ruthenium plaque radiotherapy for uveal melanoma. A meta-analysis of studies including 1,066 patients. Acta Ophthalmol Scand 1999; 77: 414-417.

8. Robertson DM. Changing concepts in the management of choroidal melanoma. Am J Ophthalmol 2003; 136: 161-170.

9. Singh AD, Topham A. Survival rates with uveal melanoma in the United States: 1973-1997. Ophthalmology 2003; 110: 962-965.

10. Singh AD, Rennie IG, Kivela $\mathrm{T}$ et al. The ZimmermanMcLean-Foster hypothesis: 25 years later. $\mathrm{Br}$ J Ophthalmol 2004; 88: 962-967.

11. Bergman L, Nilsson B, Lundell G et al. Ruthenium brachytherapy for uveal melanoma, 1979-2003: survival and functional outcomes in the Swedish population. Ophthalmology 2005; 112: 834-840.

12. Damato B, Patel I, Campbell IR et al. Local tumor control after 106Ru brachytherapy of choroidal melanoma. Int J Radiat Oncol Biol Phys 2005; 63: 385-391.

13. Marconi DG, de Castro DG, Rebouças LM et al. Tumor control, eye preservation, and visual outcomes of ruthenium plaque brachytherapy for choroidal melanoma. Brachytherapy 2013; 12: 235-239.

14. Verschueren KM, Creutzberg CL, Schalij-Delfos NE et al. Long-term outcomes of eye-conserving treatment with Ruthenium (106) brachytherapy for choroidal melanoma. Radiother Oncol 2010; 95: 332-338.

15. Naseripour M, Jaberi R, Sedaghat A et al. Ruthenium-106 brachytherapy for thick uveal melanoma: reappraisal of apex and base dose radiation and dose rate. J Contemp Brachytherapy 2016; 8: 66-73.

16. Wilkinson DA, Kolar M, Fleming PA et al. Dosimetric comparison of $106 \mathrm{Ru}$ and 125I plaques for treatment of shallow ( $<$ or $=5 \mathrm{~mm}$ ) choroidal melanoma lesions. Br J Radiol 2008; 81: 784-789.

17. Wen JC, Oliver SC, McCannel TA. Ocular complications following I-125 brachytherapy for choroidal melanoma. Eye (Lond) 2009; 23: 1254-1268.

18. Summanen $\mathrm{P}$, Immonen $\mathrm{I}$, Kivela $\mathrm{T}$ et al. Radiation related complications after ruthenium plaque radiotherapy of uveal melanoma. Br J Radiol 1996; 80: 732-739. 
19. Takiar V, Voong KR, Gombos DS et al. A choice of radionuclide: Comparative outcomes and toxicity of ruthenium-106 and iodine-125 in the definitive treatment of uveal melanoma. Pract Radiat Ocol 2015; 5: 169-176.

20. Gupta A, Muecke JS. Treatment of radiation maculopathy with intravitreal injection of bevacizumab (Avastin). Retina 2008; 28: 964-968.

21. Reichstein D. Current treatments and preventive strategies for radiation retinopathy. Curr Opin Ophthalmol 2015; 26: 157-166.

22. Valentini V, Maurizi F, Tagliaferri L et al. Spider: managing clinical data of cancer patients treated through a multidisciplinary approach by a palm based system. Ital J Publ Health 2008; 5: 154-164.

23. Tagliaferri L, Kovács G, Autorino R et al. ENT COBRA (Consortium for Brachytherapy Data Analysis): interdisciplinary standardized data collection system for head and neck patients treated with interventional radiotherapy (brachytherapy). J Contemp Brachytherapy 2016; 8: 336-343.

24. Tagliaferri L, Pagliara MM, Boldrini L et al. INTERACTS (INTErventional Radiotherapy ACtive Teaching School) guidelines for quality assurance in choroidal melanoma interventional radiotherapy (brachytherapy) procedures. J Contemp Brachytherapy 2017; 93: 287-295.

25. Astrahan MA, Luxton G, Jozsef G, et al. An interactive treatment planning system for ophthalmic plaque radiotherapy. Int J Radiat Oncol Biol Phys 1990; 18: 679-687.

26. Kaplan E, Meier P. Nonparametric estimation from incomplete observations. JAMA 1958; 53: 457-481.

27. Bozdogan H. Model selection and Akaike's Information Criterion (AIC): The general theory and its analytical extensions. Psychometrika 1987; 52: 345-370.

28. Steyerberg EW. Clinical Predictions Models. Springer-Verlag, New York 2009; 1431-1488

29. Schoenfeld D. Partial residuals for the proportional hazards regression model. Biometrika 1982; 69: 239-241.

30. Collins GS, Reitsma JM, Altman DG et al. Transparent reporting of a multivariable prediction model for individual prognosis or diagnosis (TRIPOD): the TRIPOD statement. $\mathrm{Br}$ J Surg 2015; 102: 148-158.

31. R Core Team (2016). R: a language and environment for statistical computing. $\mathrm{R}$ foundation for statistical computing, Vienna, Austria. Available at: https://www.R-project.org/.

32. Edge SB, Compton CC. The American Joint Committee on Cancer: the 7th Edition of the AJCC Cancer Staging Manual and the Future of TNM. Ann Surg Oncol 2010; 17: 1471-1474.

33. Krema H, Xu W, Payne D, et al. Factors predictive of radiation retinopathy post 125 Iodine brachytherapy for uveal melanoma. Can J Ophthalmol 2011; 46: 158-163.

34. Aziz HA, Singh N, Bena J et al. Vision Loss Following Episcleral Brachytherapy for Uveal Melanoma: Development of a Vision Prognostication Tool. JAMA Ophthalmol 2016; 134: 615-620.

35. Khan N, Khan MK, Bena J, et al. Plaque brachytherapy for uveal melanoma: a vision prognostication model. Int J Radiat Oncol Biol Phys 2012; 84: 285-290.

36. Puusaari I, Heikkonen J, Kivelä T. Ocular complications after iodine brachytherapy for large uveal melanomas. Ophthalmology 2004; 111: 1768-1777.

37. Blasi MA, Laguardia M, Tagliaferri L et al. Brachytherapy alone or with neoadjuvant photodynamic therapy for amelanotic choroidal melanoma: Functional Outcomes and Local Tumor Control. Retina 2016; 36: 2205-2212.

38. Gupta A, Muecke JS. Treatment of radiation maculopathy with intravitreal injection of bevacizumab (Avastin). Retina 2008; 28: 964-968. 